\title{
GEOREFERENCIACIÓN Y ESTUDIO DE LOS ÓRDENES DE LAS CLASES INSECTA Y COLLEMBOLA EN EL SENDERO QUISHUAR (Área Nacional de Recreación El Boliche)
}

GEOREFERENCING AND STUDY OF ORDER OF INSECTA AND COLLEMBOLA Classes in the Quishuar trail (El BOLICHE NATIONAL RESERVE)

\author{
Ondina Landázuri, Fabián Bersosa, Alex Segovia, Byron Tarabata, Patsy Prieto, \\ Rodrigo Tufiño, Gustavo Navas
}

Centro de Investigación y Modelamiento Ambiental, CIMA-UPS, Universidad Politécnica Salesiana, Rumichaca y av. Morán Valverde s/n. Telef. (593 2) 3962800 Ext. 2352, Quito, Ecuador

Autor para correspondencia: olandazuri@ups.edu.ec

Manuscrito recibido el 18 de abril de 2013. Aceptado, tras revisión, el 16 de julio de 2013.

\section{Resumen}

Con el objetivo de determinar los órdenes de las clases Insecta y Collembola, presentes en el sendero Quishuar, el 15 de septiembre de 2012, se efectuó un muestreo preliminar de los insectos y collembolos presentes en el sendero Quishuar del Área Nacional de Recreación El Boliche. Para el efecto se realizó el registro en nueve puntos de muestreo distanciados cada uno de ellos por $100 \mathrm{~m}$, donde se realizaron observaciones por 10 minutos tanto en el lado derecho como en el izquierdo del sendero en un área de $1 \mathrm{~m}^{2}$. Los insectos y collembolos fueron extraídos manualmente para luego ser liberados. Los datos registrados fueron organizados y analizados en una matriz usando los programas Excel $®$ y Past versión 2.4. La georeferenciación y los puntos trazas fueron incorporados a una herramienta Gis de Software Libre, Quantum GIS (QGis), versión 1.7.4. Los resultados registraron siete órdenes de insectos y un orden no identificado de collembola en los estratos de suelo, herbáceo y arbustivo. Los órdenes con mayor frecuencia fueron: Díptera (Insecto) y un de la clase Collembola. A través de un estudio de similitudes de los puntos de muestreo, se encontró que el mayor porcentaje de similitud se presenta entre los estratos arbustivos y suelo con el $44,4 \%$.

Palabras claves: georeferenciación, órdenes, clase Insecta, clase Collembola, El Boliche, sendero Quishuar.

\section{Abstract}

In order to determine the orders of insecta and collembola classes in the Quishuar trail, the 15th September 2012, a preliminary sample collection was done in El Boliche National Reserve. For this purpose, nine sampling spots were selected with a separation distance of $100 \mathrm{~m}$ and an area of 1 square meter; in each spot a 10 minutes observation was performed in both sides of the spot and the number of insects and collembolos was registered. After a manual recollection insects, they were released again. Excel and Past version 2.4 packages was used to analyze the obtained data. Georeferencing was performed with a GPS garmin GPSMAP 78s and the spot position was included using the open software Quantum GIS (Qgis), a GIS software tool Version 1.7.4. The results shown 7 orders of insects and one non identified collembola order inside the soil, herbaceous and bush substrates. The orders observed with higher frequency were Diptera (insecta) and one of collembola classes. The study of similitudes of sampling spots revealed that the higher percent of similitude is observed in soil and bush substrates.

Keywords: georeferencing, orders, insecta class, collembola class, El Boliche National Reserve, Quishuar trail.

Forma sugerida de citar: Landázuri, O., F. Bersosa, A. Segovia, B. Tarabata, P. Prieto, R. Tufiño y G. Navas. 2013. Georeferenciación y estudio de los órdenes de las clases insecta y collembola en el sendero Quishuar (Área Nacional de Recreación El Boliche). La Granja. Vol. 17(1): 25-35. ISSN: 1390-3799. 


\section{Introducción}

En Ecuador existen dos áreas de recreación, las cuales poseen una extensión total de $26,83 \mathrm{Km}^{2}$. El Área Nacional de Recreación El Boliche cuenta con $4 \mathrm{Km}^{2}$. Este espacio es uno de los más representativos en cuanto a flujo de visitantes a nivel nacional $(5,46 \%)$ y se encuentra cerca al Parque Nacional Cotopaxi. Sus suelos son franco-arenosos-húmedos y es una zona rica en recursos hídricos, lo que ha favorecido al desarrollo de flora y fauna en el lugar; los bancos genéticos de flora (suministros de semillas) y fauna están distribuidos en forma aislada en toda el área, específicamente en las quebradas. Existen especies arbóreas, arbustivas, herbáceas, trepadoras, epífitas y grandes cantidades de musgos, formando en conjunto pequeños relictos de gran belleza paisajística (Bastidas y Medina, 2010).

Acorde con la propuesta de clasificación vegetal de (Sierra), el área protegida se encuentra en el Sector Centro de la Cordillera Occidental y presenta tres formaciones vegetales: Bosque Siempreverde montano alto (3000-3400 msnm), páramo de herbáceas (3400-4000 msnm) y páramo de Almohadillas (4000-4500 msnm).

La fauna del lugar pertenece al piso zoogeográfico altoandino; sin embargo, no se cuenta con datos actuales del número de especies faunísticas en el Área de Recreación (ECOLAP y MAE, 2007).

Asimismo, el Área cuenta con un mirador, la Planicie de Sunfana y senderos como Romerillos y Quishuar, constituyéndose éste último en el sitio de estudio (Figura 2).

El recorrido a pie por el sendero Quishuar toma aproximadamente 40 minutos (ECOLAP y MAE, 2007); el trayecto es a través del bosque de Pinus radiata (pino) y especies forestales andinas y subandinas como, Buddleja incana (quishuar), Oreopanax sp. (pumamaqui), Vallea stipularis (sacha capulí), entre otras (Andrade, 2010). El suelo en su mayoría está cubierto por gran cantidad de musgo, hojarasca y pocas herbáceas pequeñas (Figura 1).

En lo referente al aspecto taxonómico del estudio, en el pasado dentro del Phylum Arthropoda, los insectos fueron clasificados en dos subclases: Apterigota y Pterigota; sin embargo nuevas evidencias filogénicas han establecido una separación de estos taxones y el establecimiento de la superclase Hexápoda con dos clases independientes: Insecta y Collembola (Bach de la Roca et al., 1999).

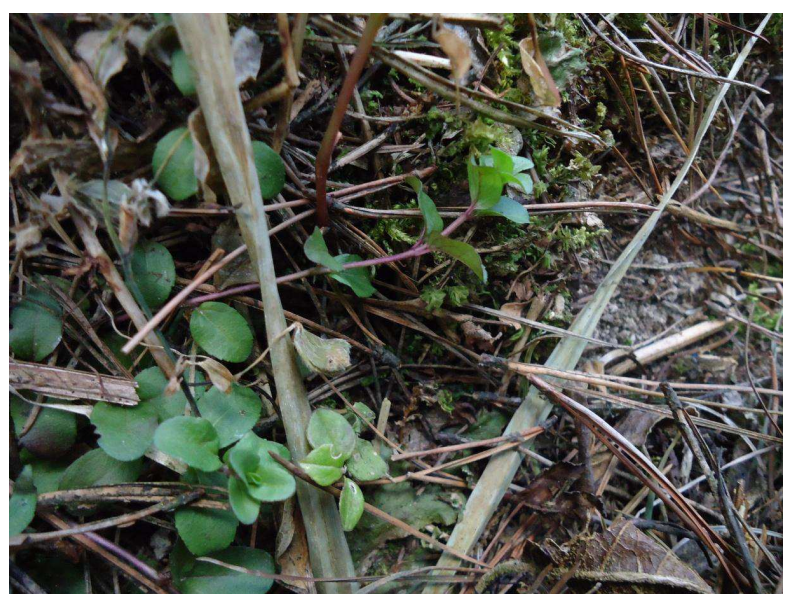

Figura 1. Cobertura típica del suelo compuesta por musgo, hojarasca y hierbas pequeñas en el sendero Quishuar. Fuente: Byron Tarabata

Sin importar el taxón en el que se encuentren los grupos motivo de estudio, en general los artrópodos cumplen un sinnúmero de funciones en el ecosistema en favor del ambiente y del ser humano; sin embargo dentro de este grupo existen organismos que han causado graves daños en algunos componentes bióticos del ecosistema (Melic), generalmente por acción del ser humano se han transformado en plagas (Owen, 2000). Por tanto conocer los órdenes presentes en un área dada es el primer paso que abre la posibilidad de continuar con estudios más minuciosos sobre el nicho real de las especies en forma más puntual.

De esta manera, se determinarán los sitios donde los órdenes de las clases Insecta y Collembola se encuentren presentes durante el muestreo a lo largo del sendero Quishuar en el Área Nacional de Recreación el Boliche, así como su frecuencia en los tres estratos (suelo, herbáceo y arbustivo).

A futuro esta información podrá estar disponible para quien desee ubicar los sitios estudiados, ampliar esta investigación o como fuente de consulta para cualquier usuario. 


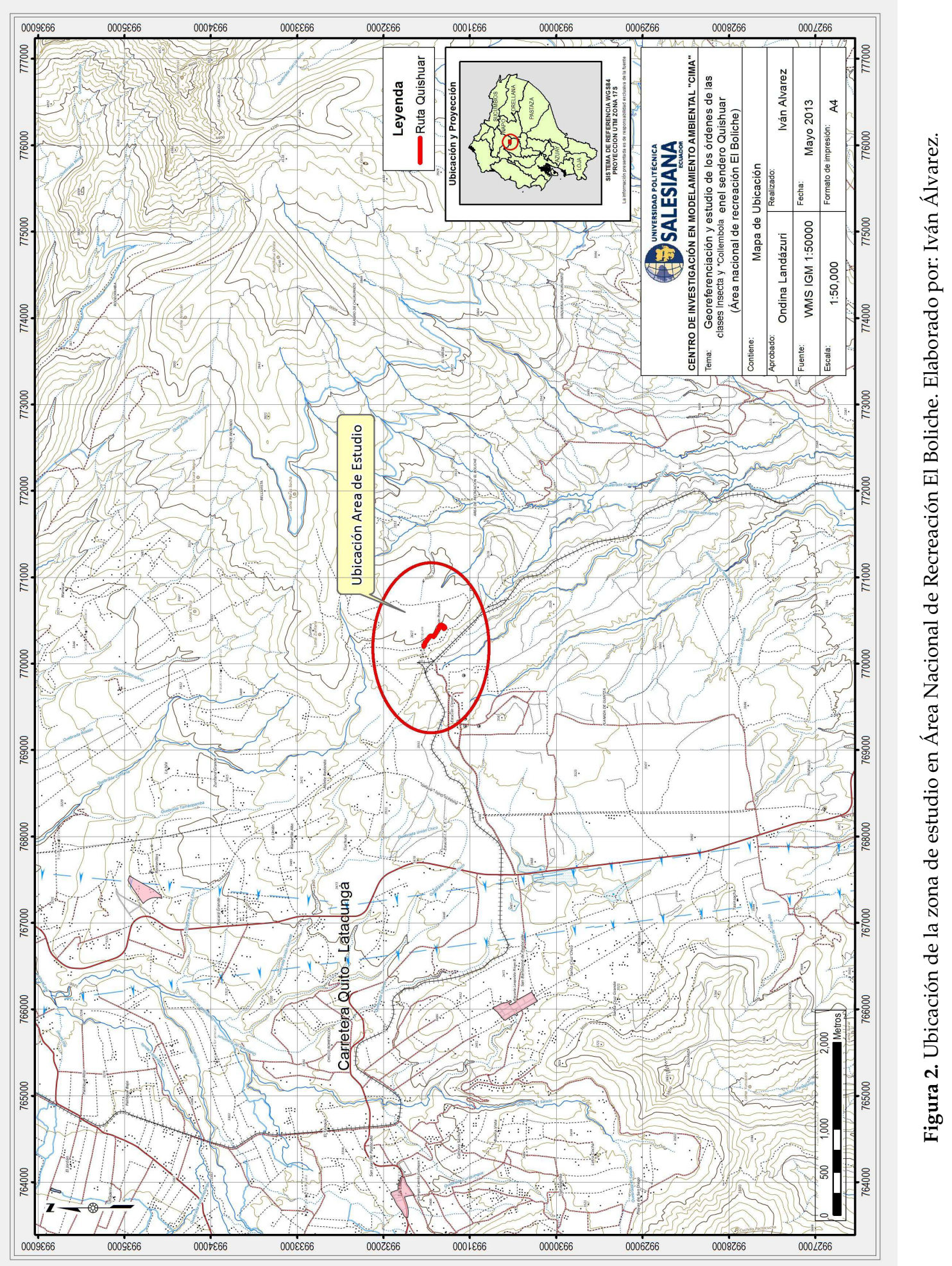

LA GRANJA, Revista de Ciencias de la Vida, 17(1) 2013: 25-35. 


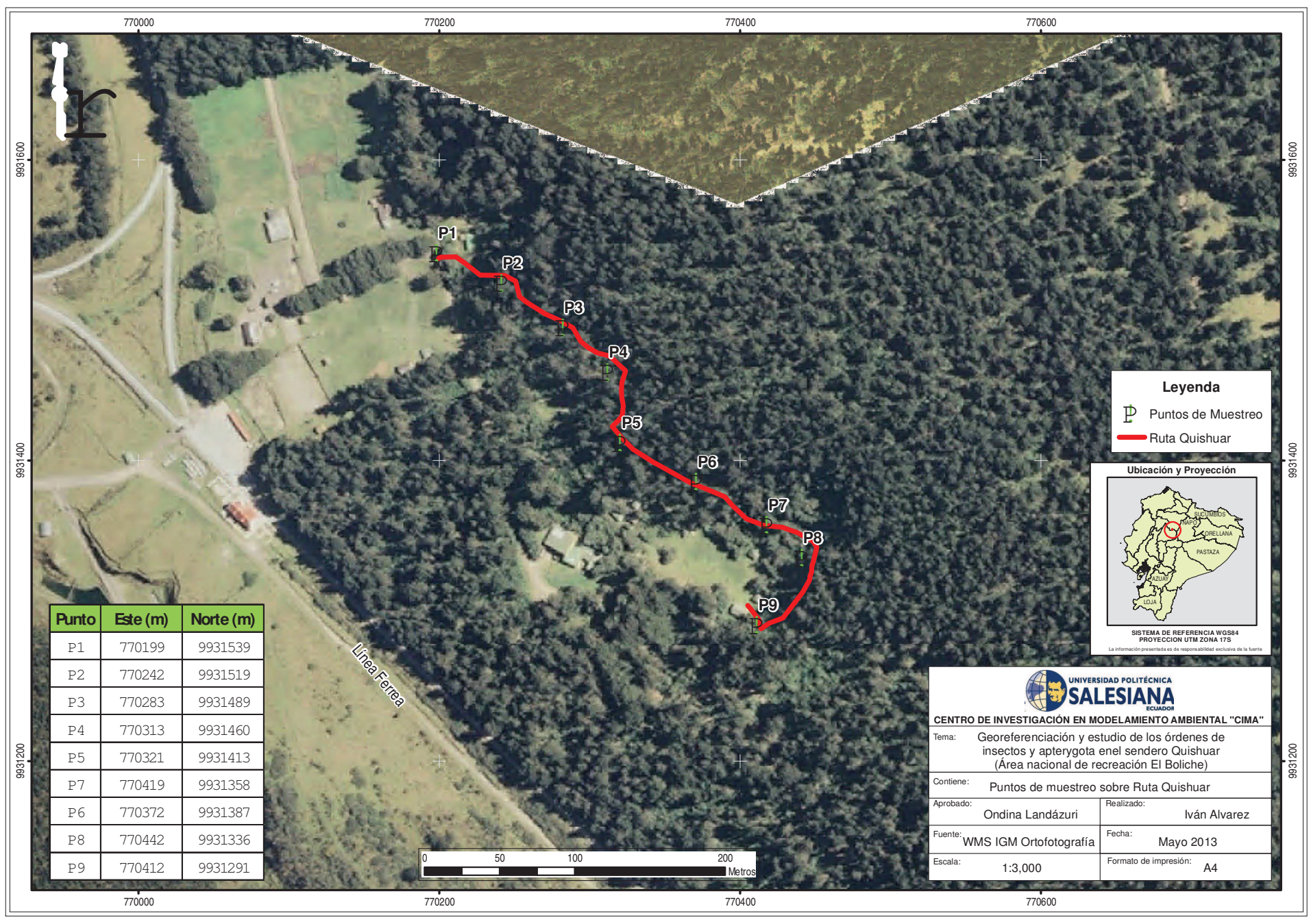

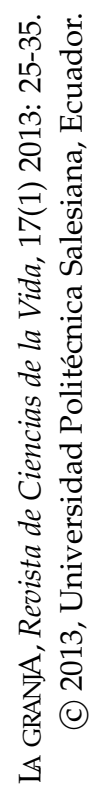

Figura 3. Puntos de muestreo de los órdenes de las clases Insecta y Collembola (clase) en el sendero Quishuar; el símbolo p representa el punto de muestreo y los números del 1 al 9 indican la secuencia; los dos símbolos unidos indican las ubicación de los nueve puntos de muestreo georeferenciado. Elaborado por: Iván Álvarez. 
En cuanto a la georeferenciación, su importancia radica es ser una herramienta tecnológica que permite ubicar los sitios estudiados y que es utilizada en un amplia gama de investigaciones en ciencias biológicas y humanas (Sletto et al., 2010; Navas y Prieto, 2011) gracias al uso del GPS, (Sistema de Posicionamiento Global) se logran mapas con gran exactitud. Asimismo, los sistemas de información geográfica son herramientas de visualización sobre patrones, relaciones o tendencias gracias a que son capaces de manejar funciones de análisis muy avanzados (Pinde \& Jiulin, 2011).

Estas nuevas funcionalidades, unidas al uso progresivo de Internet, han hecho que exista una migración casi natural desde los sistemas de información geográfica de escritorio a las aplicaciones de Sistema de Información Geográfica (SIG) en la Web, Infraestructuras de Datos Espaciales (IDES) o geoportales (Carr y Rich, 2008), se entiende por geoportales a los sitios web donde un usuario puede buscar información o recursos geográficos, WMS (Web Map Service) utilizando un navegador, datos que son publicados por un proveedor quien difunde la información geográfica y los metadatos asociados.

\section{Materiales y Métodos}

\subsection{Muestreo}

El trabajo se efectuó el 15 de septiembre de 2012, de 8:00 a 12:00 AM, en el sendero Quishuar ubicado en la Área Nacional de Recreación El Boliche, con una altitud de 3584 m.s.n.m hasta 3600 m.s.n.m.

Para realizar el muestreo de los órdenes de las clase Insecta y Collembola, se efectúo un transecto de $950 \mathrm{~m}$ de extensión, en el que cada 100 metros se estableció un sitio de muestreo de $1 \mathrm{~m}^{2}$, con observaciones de 10 minutos en cada metro cuadrado, tanto a la izquierda (iz) como a la derecha (d) del sendero, se logró un total de nueve puntos de muestreo (p) (Figura 3).

Los especímenes reportados no fueron colectados y su identificación fue a nivel de orden, para certificar las identificaciones preliminares se fotografiaron los especímenes observados mediante una cámara fotográfica Sony Cyber-shot DSC-W530. Ver Anexo 1, registro fotográfico.

Los órdenes reportados fueron analizados a nivel de riqueza, frecuencia y similitud de Jacard mediante el programa Past versión 2.4 (Hammer et al., 2001), el cual se basa en la presencia y ausencia de los órdenes en los estratos estudiados; así se obtuvo valores de 0 a 1 ; el valor se transforma en porcentaje e indica la similitud entre los estratos.

\subsection{Georeferenciación}

La georeferenciación del sitio de estudio se efectuó mediante el uso de un GPS, modelo GPSMAP 78s, que genera directamente formatos gpx, este es un estándar para exportar o migrar a cualquier plataforma GIS o CAD (Computer Aided Design), esto permite separar cada grupo de datos como archivos diferentes, como puntos gps y trazas, que se incorporan a formatos GIS, para la creación de capas para los puntos gps de muestreo y otro para el recorrido total efectuado en el estudio, que fueron incorporados a una herramienta GIS de Software Libre, QuantumGIS (QGis), versión 1.7.4. Desde este se grabó en formato Shape, el cual es uno de los formatos GIS más comunes, así como también se exportó a la base de datos relacional, para el caso concreto PostgreSQL 9.1 y dentro de este la base de datos especializada para el manejo de datos espaciales PostGis.

\section{Resultados y discusión}

Se registraron un total de 41 individuos, pertenecientes a las clases Insecta y Collembola agrupados en ocho órdenes. El orden con mayor frecuencia fue Díptera $(n=8)$ con un porcentaje de frecuencia del $89 \%$ y la clase Collembola $(n=7)$, con un orden no determinado con un porcentaje de $78 \%$ de presencia en los puntos estudiados (Figura 4).

A nivel de riqueza de órdenes el punto 8 fue el sitio más diverso con 6 órdenes, a continuación los sitios 2 y 6 con 5 órdenes, los sitios 1,5 y 9 con 4 órdenes y finalmente los sitios 3, 4 y 7 tan solo tuvieron 3 órdenes presentes (Figura 5). 


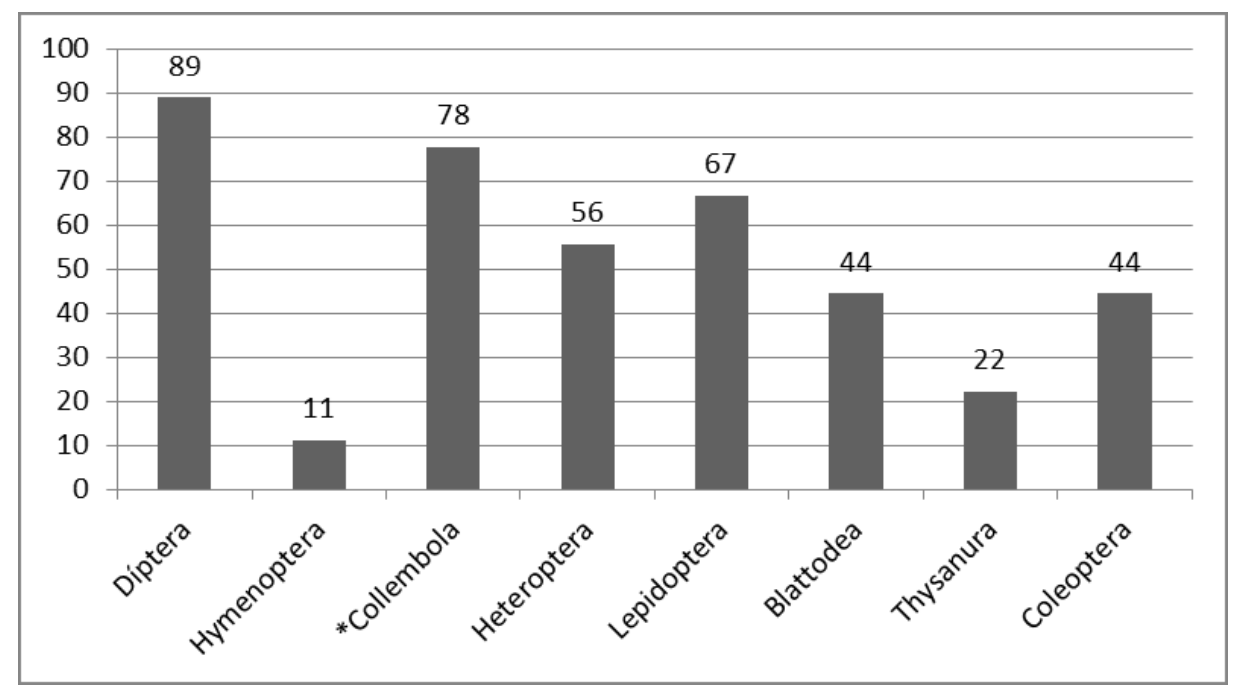

Figura 4. Porcentajes de las Frecuencias de los órdenes de las clase Insecta y *Collembola (con un orden no determinado) reportados en los puntos de muestreo del Sendero Quishuar.

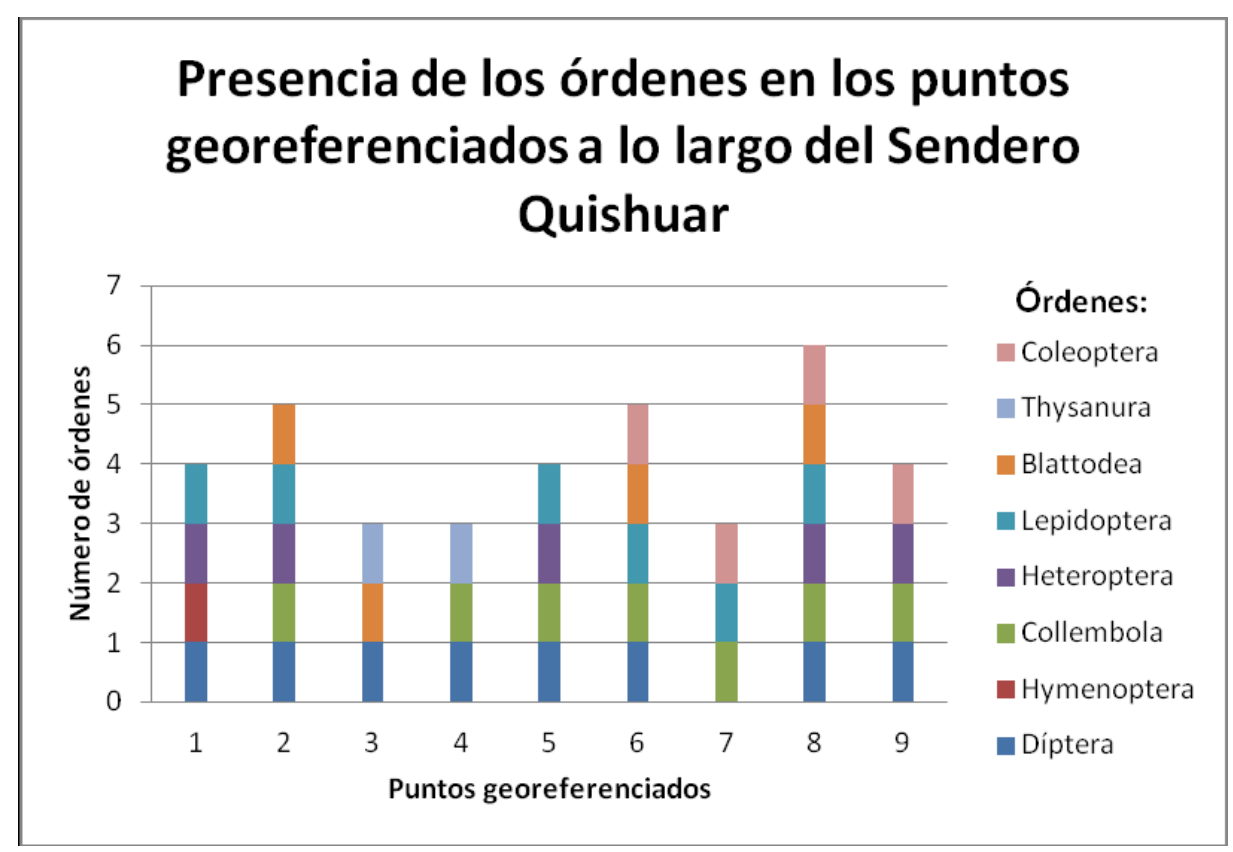

Figura 5. Figura 5. Riqueza de órdenes de las clases Insecta y *Collembola (orden no determinado) en los nueve sitios (puntos) de muestreo a lo largo del sendero Quishuar. 
Tabla 1. Presencia de los órdenes de las clases Insecta y Collembola (orden no determinado) en los estratos estudiados.

\begin{tabular}{llccc}
\hline CLASE & ORDEN & arbustivo & herbáceo & suelo \\
\hline Collembola & No determinado & $\mathrm{X}$ & & $\mathrm{X}$ \\
Insecta & Blattodea & & & $\mathrm{X}$ \\
& Coleoptera & & & $\mathrm{X}$ \\
& Diptera & $\mathrm{X}$ & $\mathrm{X}$ & $\mathrm{X}$ \\
& Heteroptera & $\mathrm{X}$ & $\mathrm{X}$ & $\mathrm{X}$ \\
& Hymenoptera & & & $\mathrm{X}$ \\
& Lepidoptera & & $\mathrm{X}$ & $\mathrm{X}$ \\
& Thysanura & & & $\mathrm{X}$ \\
\hline & & 3 & 3 & 8 \\
\hline
\end{tabular}

A nivel de estratos se encontraron 8 órdenes (Díptera, Hymenoptera, Heteroptera, Lepidoptera, Blattodea, Thysanura, Coleoptera y el orden no determinado de la Clase Collembola) en el estrato suelo, 3 órdenes (Díptera, Heteroptera y Lepidoptera) en el estrato herbáceo y 3 órdenes (Díptera, Heteróptera y el orden no determinado de la Clase Collembola) en el estrato arbustivo. Ver Tabla 1.

El mayor porcentaje de similitud se encontró entre los estratos arbustivos y herbáceo con el 50\% y el menor porcentaje de similitud se encontró entre los estratos herbáceo -suelo y arbustivo- suelo con el $37 \%$ en ambos casos. Ver Tabla 2.

Tabla 2. Porcentaje de similitud por órdenes y estratos

\begin{tabular}{lccc}
\hline & Arbustivo & Herbáceo & Suelo \\
\hline Arbustivo & $100 \%$ & $50 \%$ & $37,5 \%$ \\
Herbáceo & $50 \%$ & $100 \%$ & $37,5 \%$ \\
Suelo & $37,5 \%$ & $37,5 \%$ & $100 \%$ \\
\hline
\end{tabular}

Este estudio sobre la fauna de insectos y collembolos en el Sendero Quishuar es el primero en su tipo en ésta área, lo que ha permitido tener una visión general acerca de la presencia de ocho órdenes, con 41 individuos encontrados a lo largo del sendero; los órdenes más frecuentes son: Díptera (Insecta) y un orden no determinado de la clase Collembola.

El muestreo permitió conocer preliminarmente el número de órdenes que conforman la comunidad en los diferentes puntos determinados; se concluye que solo en tres puntos: tres, cuatro y siete, la rique- za de órdenes disminuye a tres órdenes que representa a la comunidad de insectos y collembolos.

Díptera y Heteroptera fueron los órdenes de la clase Insecta presentes en los tres estratos, mas se encontró el orden Díptera en mayor porcentaje.

Por otro lado, el estrato suelo, es donde el mayor número de órdenes se hace presente, probablemente porque existe mayor superficie con alimento y protección para los insectos y collembolos presentes o, tal vez, porque es el nicho real de los ocho órdenes encontrados en el sendero. A continuación se presentan los estratos herbáceo y arbustivo con igual número de órdenes, compartiendo dos órdenes en común Díptera y Heteroptera (Figura 6).

\section{Conclusiones y recomendacio- nes}

Se encontraron 41 individuos pertenecientes a siete órdenes de la clase Insecta y un orden no determinado de la clase Collembola en los 9 puntos de estudio. Los órdenes Díptera de la clase Insecta y un orden no determinando de la clase Collembola, son los taxones más representativos de los ocho encontrados a lo largo del sendero estudiado. La riqueza de órdenes se mantiene a lo largo del sendero, excepto en tres puntos del muestreo (3, 4 y 7$)$.

El estrato suelo presenta el mayor número de órdenes de insectos, le siguen a continuación los estratos suelo y herbáceo con el mismo número de órdenes. 


\section{Presencia de los órdenes por estratos}

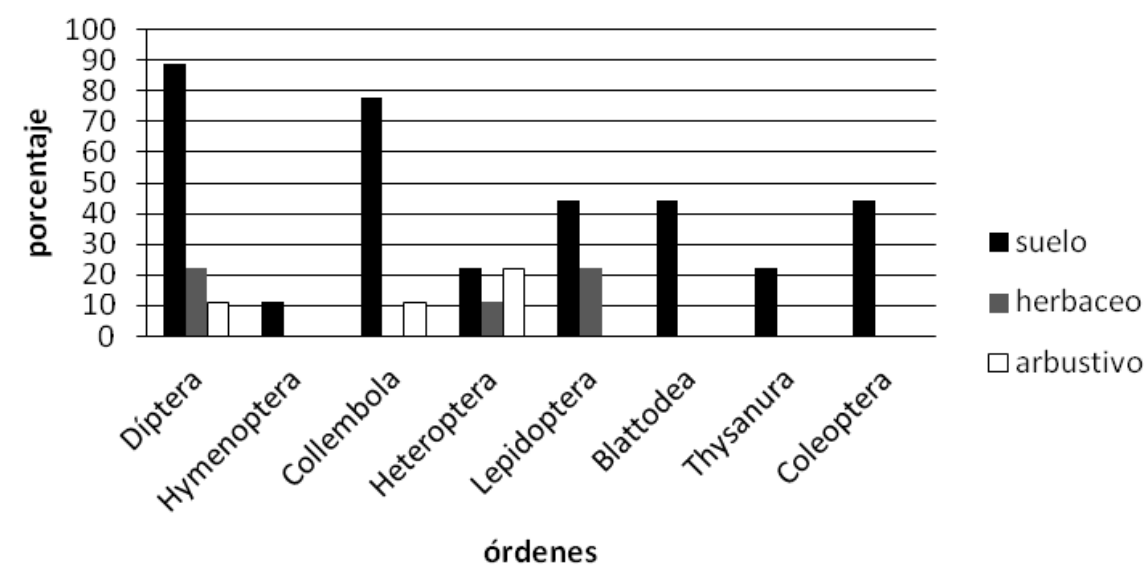

Figura 6. Porcentaje de los órdenes de las clases Insecta y *Collembola (orden no determinado) presentes en los estratos suelo, herbáceo y arbustivo en el sendero Quishuar.

De esta manera, el presente estudio muestra un análisis preliminar de los órdenes de las clases Insecta y Collembola presentes en el Sendero Quishuar y, por lo tanto, en la zona correspondiente al Área Nacional de Recreación El Boliche. Nuevas investigaciones basadas en los resultados presentados, como aquellas que generen un inventario más detallado de las especies presentes, sus nichos e interacción ecológica, podrían determinar de una manera más precisa la diversidad entomológica de la zona.

\section{Agradecimientos}

La presente investigación ha sido financiada por la "Cuarta Convocatoria de Proyectos de Investigación de la Universidad Politécnica Salesiana". Asimismo, los autores agradecen al Ministerio del Ambiente de Ecuador, a la Dirección del Ambiente de Cotopaxi por facilitarnos los permisos respectivos para realizar esta investigación y al personal del Área Nacional de Recreación El Boliche por las facilidades prestadas para la presente investigación. Se agradece también a Iván Âlvarez por la elaboración de los mapas presentados en este estudio y a Sheila Serrano por su colaboración en la discusión de los resultados presentados.

\section{Referencias}

Andrade, A. 2010. Plan de marketing turístico para el Área nacional de recreación el boliche, ubicada en la parroquia de mulaló, provincia de cotopaxi. Tesis Doctoral, Universidad Tecnológica Equinoccial.

Bach de la Roca, C., M. Gaju-Richard y A. CompteStart. 1999. Evolución y filogenia de artrópoda. S.E.A.(26): 379-395.

Bastidas, D. y P. Medina. 2010. Estimación de la Densidad Poblacional del Ecuador Continental. Unidad de Análisis de la Información Estadística, Instituto Nacional de Estadística y Censos, Juan Larrea N15-36 y José Riofrío Quito, Ecuador.

Carr, T. y J. Rich, P. Nd Bartley. 2008. The natcarb geoportal. Journal of Map \& Geography, págs. 131-147.

ECOLAP y MAE. 2007. Guía del patrimonio de Áreas naturales protegidas del ecuador. IGM. Quito, Ecuador, ECOFUND, FAN, DarwinNet, URL 〈http://www.gstalliance.net /ecuador/index2.php?option=com_docman $\&$ task $=$ doc_view\&gid $=78 \&$ Itemid $=207\rangle$, consulta: 17 octubre de 2012. 
Hammer, O., D. Harper y P. Ryan. 2001. Past: Paleontological stadistics software package for education and data analysis. palaentologia electrónica. URL /http://palaeo-electronica.org/2001_1/past /past.pdf), consulta: 20 de noviembre de 2012.

Melic, A. Los artrópodos y el hombre. Boletín de la S.E.A., No. 20: 5-13, URL 〈http://www.sea-entomologia.org $\rangle$, consulta 20 de noviembre de 2012.

Navas, G. y P. Prieto. 2011. Geoportales en el ecuador. La Granja, 14(2): 58-64.

Owen, O. 2000. Conservación de Recursos Naturales. segunda edición edición,
URL 〈http://books.google.es/books?id=0Z _KmG0yOvEC\&pg=PA515\&dq=origen + de+las + plagas\&hl=es\&sa $=$ X\&ei=9tIOUZauNJLr0QHru IGYBA\&ved=0CEsQ6AEwBjgK $\rangle$, consulta 20 de noviembre de 2012., págs. 515-516.

Pinde \& Jiulin. 2011. Web gis. California: Esri Press.

Sierra, R. Propuesta preliminar de un sistema de clasificación de vegetación para el Ecuador continental. Quito.

Sletto, B. S. M., S. Strange y R. Donoso. 2010. El rincón de los olvidados: Participatory gis, experiential learning and critical pedagogy in santo domingo. Journal of Latin American Geography, 9(3): 111-135. 


\section{Anexos}

\section{Anexo 1}

Registro fotográfico de órdenes de las clases Insecta y Collembola (clase) registrados en el sendero Quishuar, Área Nacional de Recreación El Boliche, Fuente: Byron Tarabata.

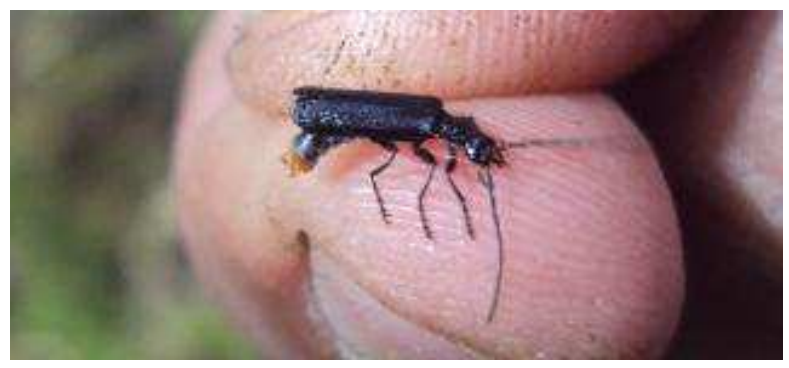

Coleoptera

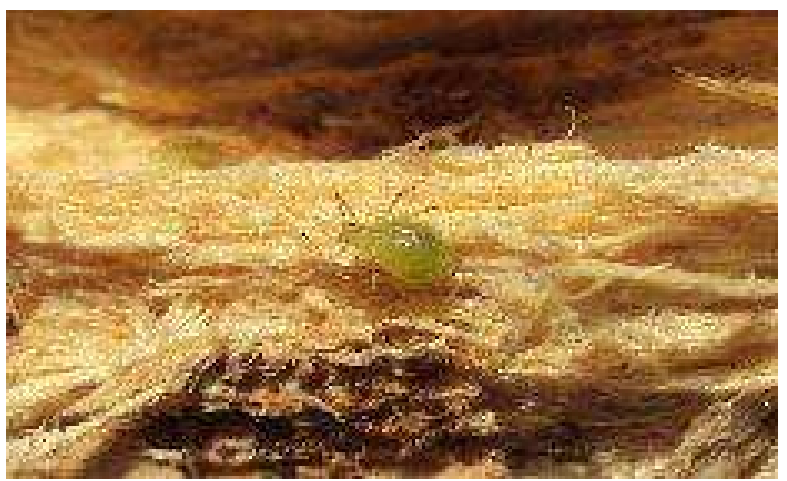

Heteroptera (Hemiptero)

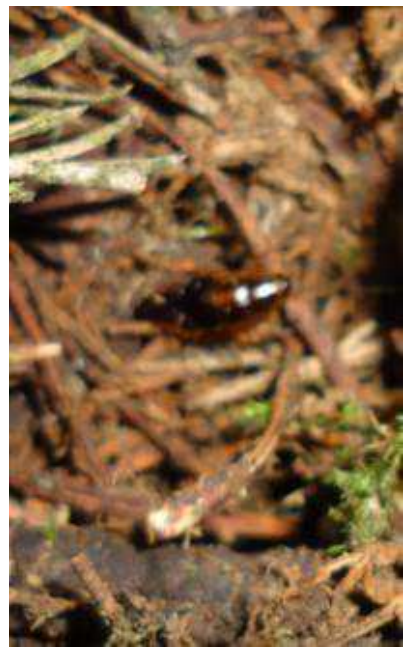

Blattoidea

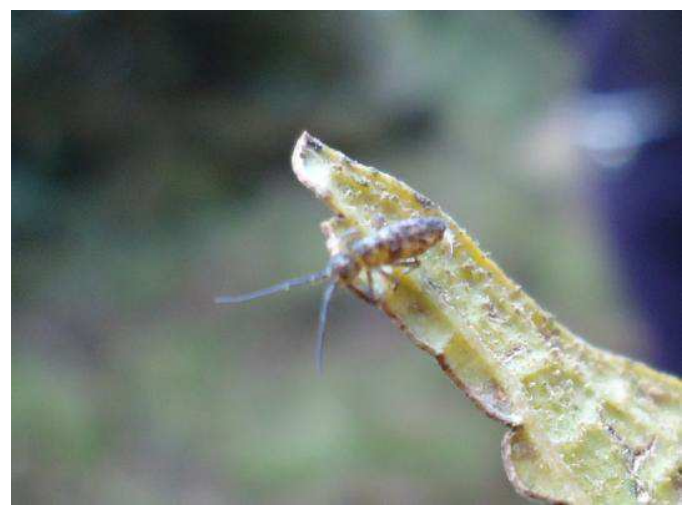

Collembola

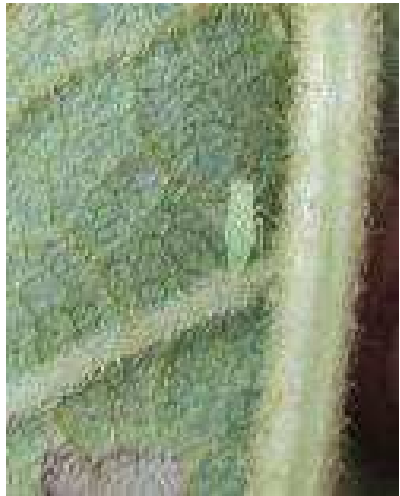

Heteroptera (Homoptero)

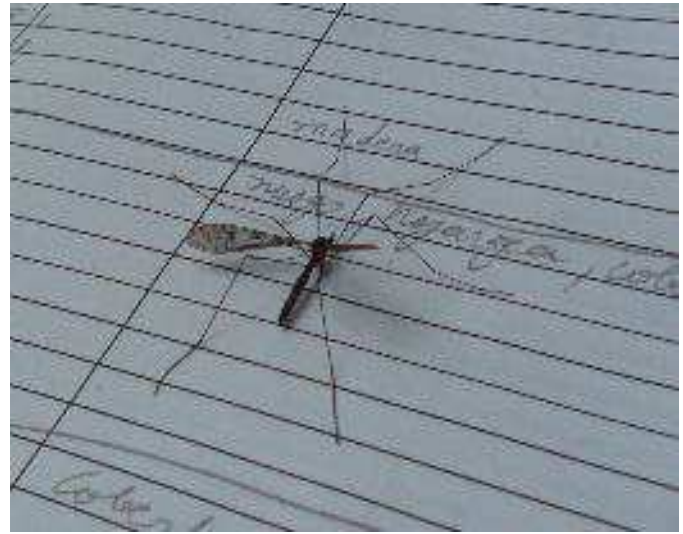

Díptera (Tipulidae) 


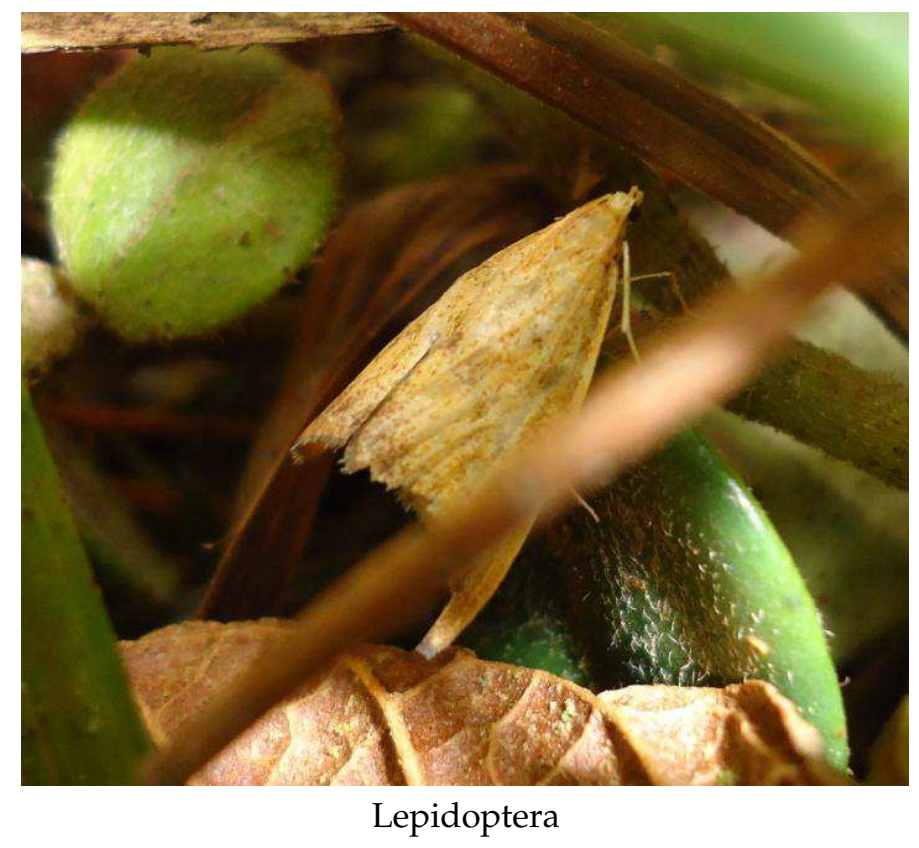

LA GRANJA, Revista de Ciencias de la Vida, 17(1) 2013: 25-35.

(C) 2013, Universidad Politécnica Salesiana, Ecuador. 